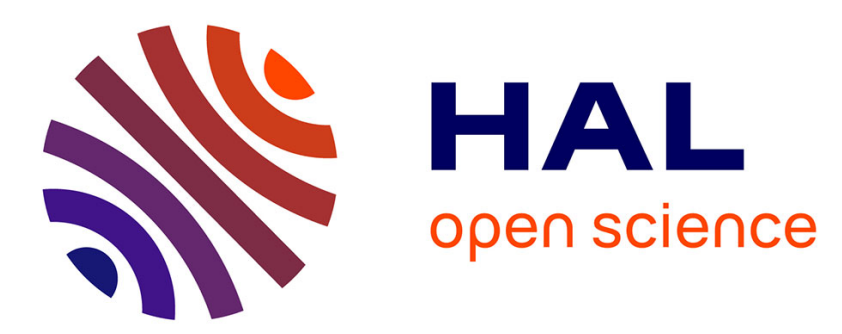

\title{
Valuing life: experimental evidence using sensitivity to rare events
}

Olivier Chanel, Graciela Chichilnisky

\section{To cite this version:}

Olivier Chanel, Graciela Chichilnisky. Valuing life: experimental evidence using sensitivity to rare events. 2011. halshs-00651163

\section{HAL Id: halshs-00651163 \\ https://shs.hal.science/halshs-00651163}

Preprint submitted on 13 Dec 2011

HAL is a multi-disciplinary open access archive for the deposit and dissemination of scientific research documents, whether they are published or not. The documents may come from teaching and research institutions in France or abroad, or from public or private research centers.
L'archive ouverte pluridisciplinaire HAL, est destinée au dépôt et à la diffusion de documents scientifiques de niveau recherche, publiés ou non, émanant des établissements d'enseignement et de recherche français ou étrangers, des laboratoires publics ou privés. 


\section{GREQAM}

Groupement de Recherche en Economie Quantitative d'Aix-Marseille - UMR-CNRS 6579

Ecole des Hautes études en Sciences Sociales Universités d'Aix-Marseille II et III
Document de Travail $n^{\circ} 2011-54$

Valuing life: experimental evidence using sensitivity to rare events

Olivier Chanel Graciela Chichilnisky

December 2011 


\title{
Valuing life: experimental evidence using sensitivity to rare events
}

\author{
Olivier Chanel \\ CNRS-GREQAM-IDEP \\ 2 Rue de la Charité \\ F-13236 Marseille cedex 2 \\ France
}

\section{Graciela Chichilnisky}

Departments of Economics and of Statistics

Columbia Consortium for Risk Management

Columbia University

New York 10027

USA

Email: chichilnisky1@gmail.com

\section{Corresponding author:}

Olivier Chanel

Phone: + 33491140780

Fax: + 33491900227

Email: chanel@univmed.fr 


\begin{abstract}
Global environmental phenomena like climate change, major extinction events or flutype pandemics can have catastrophic consequences. By properly assessing the outcomes involved - especially those concerning human life - economic theory of choice under uncertainty is expected to help people take the best decision. However, the widely used expected utility theory values life in terms of the low probability of death someone would be willing to accept in order to receive extra payment. Common sense and experimental evidence refute this way of valuing life, and here we provide experimental evidence of people's unwillingness to accept a low probability of death, contrary to expected utility predictions. This work uses new axioms of choice, especially an axiom that allows extreme responses to extreme events, and the choice criterion that they imply. The implied decision criteria are a combination of expected utility with extreme responses, and seem more consistent with observations.
\end{abstract}

Keywords: Decision under risk, Value of Prevented Fatality, Expected Utility, Experiment, Catastrophic risk.

\title{
1 Introduction
}

Global environmental phenomena like climate change, major extinction events or flu-type pandemics share two characteristics: potential catastrophic consequences and a high degree of uncertainty. To determine the best decision to take in order to mitigate or avoid their harmful consequences, decision theorists use the choice under uncertainty framework, especially the widely-applied Expected Utility (EU). This then commonly assesses potential outcomes, including those affecting human life when deaths are involved. In essence, EU theory values life in terms of the low probability of death that would be acceptable in return for a given amount of money.

However, Arrow (1966) provided the following illustration of how people value their 
lives that puzzles decision economists: Most people would prefer 5 cents to 2 cents, and 2 cents to death. Does this mean that they would prefer 5 cents and a very low probability of death, to 2 cents? Kenneth Arrow famously commented that a positive response to this question would seem "outrageous at first blush". And yet the answer is 'Yes' according to the EU theory that Von Neumann-Morgenstern, he and others pioneered (Arrow, 1971). For instance, if we take a Value of a Prevented Fatality (VPF) of $\$ 5.5$ million (US-EPA, 2004), the corresponding probability of death that would be acceptable for an extra 3 cents is $5.4510^{-9}$ (i.e. $0.03 / 5.510^{6}$ ) according to EU.

Consequently, Arrow's comment is fully relevant, although at first glance it could be argued that the amounts at stake in his example are too small to make sense. But Arrow's famous example can be reworked within a simple experiment that provides much larger numerical values.

In February 1998, 64 subjects were invited to play a hypothetical game in which they could choose whether or not to swallow one pill among 1 billion $\left(10^{9}\right)$ identical ones. Only one pill contained a lethal poison that was sure to kill, all the others being harmless. The survivors (i.e. those who swallowed one of the 999,999, 999 harmless pills) received $\$ 220,000$. We easily infer the value these subjects attribute to their own life according to EU predictions. Each of the 33 subjects who answered ' $N o$ ', implicitly valued his/er own life at more than $\$ 220$ trillion $\left(220,000 / 10^{-9}\right)$. This VPF obviously contrasts with the $\$ 1.7-\$ 7$ million range usually obtained in the literature. The same game was played again by the same subjects as well as new subjects in January 2009, providing similar results as well as motivations for their (possible change in) answers.

This article examines the results of this experiment, and takes another look at Arrow's comment. The theory we present reveals that this puzzling result can be attributed to the failure of EU theory to provide an appropriate value for catastrophic events such as death. It is well known that EU theory has limitations and individuals have been found to 
violate its axioms in a variety of settings since the 1950's (historical examples are Allais' 1953, and Ellsberg's 1961 paradoxes). Chichilnisky (2000) showed that it underestimates our responses to rare events no matter how catastrophic they may be. This insensitivity has unintended consequences. We argue that this insensitivity, and the attendant inability to explain responses to choices where catastrophic outcomes are possible, make EU theory less appropriate to properly express rationality in these situations. A case in point is the experimental paradox presented above when valuing human life, since EU theory does not "fit" with the stated behavior of most of the subjects in the experiment.

This paper provides a theoretical framework by considering death as a 'catastrophe', namely a rare event with major consequences. Using the new axioms of choice introduced in Chichilnisky $(2000,2002)$, we derive a choice criterion that is more consistent with the experimental evidence on how people value catastrophic events such as death. We show that EU theory underestimates rare events and that this originates from the classic axiom of continuity (Monotone Continuity, defined in Arrow, 1971) which implies that rational behavior involves insensitivity to rare events with major consequences like death. We replace the axiom of continuity by an alternative axiom of sensitivity to rare events, formalizing a theory of choice under uncertainty where rare but catastrophic events (such as death) are given a treatment in symmetry with the treatment of frequent events. As a consequence, a probability can be considered low enough to make the lottery involving death acceptable; it all depends on what the other outcomes are.

This implies a different way of valuing life, one that seems more in tune with experimental evidence. First, this new way of valuing life is in keeping with evidence provided by the experiment reported below, given that age and family situation appear to affect the way subjects change their decisions about whether or not to take action impacting the value of their lives. More generally, it may explain why in some experiments people appear to give unrealistically high numerical values to life that are not consistent with the empirical 
evidence about how they choose occupations, for instance. Second, this new way of valuing life is in keeping with evidence provided by experimental psychologists, who observe that the brain reacts differently when making a decision involving rare situations inspiring extreme fear (LeDoux, 1996). Overall, the proposed framework suggests an alternative way to define rational behavior when catastrophic risks are involved.

The remainder of the paper proceeds as follows. Section 2 presents the experimental evidence. Section 3 recalls recent contributions in the literature on modeling risk and catastrophic events, shows how EU theory fails to appropriately value life and proposes a solution. The final Section discusses the results and draws conclusions.

\section{Experimental evidence}

We present the results of an experiment (referred to below as the pill experiment) which twice asked a sample of subjects a question implying a trade-off between the risk of dying and a fixed amount of money, at an interval of 11 years.

\subsection{The 1998 initial pill experiment}

In February 1998, the members of a Research Center in Quantitative Economics were asked by internal e-mail (in French): "Imagine that you are offered the opportunity to play a game in which you must choose and swallow one pill out of 1 billion $\left(10^{9}\right)$ identical pills. Only one contains a lethal poison that is sure to kill you, all the other pills being ineffective. If you survive (i.e. you swallow one of the 999, 999, 999 ineffective pills), you receive a tax-free amount of $€ 152,450 .^{1}$ Are you willing to choose one pill and to swallow it?".

The value subjects attribute to their own life can be assessed using the classic utility theory of choice under uncertainty. Indeed, state-dependent models, simple single period

\footnotetext{
${ }^{1}$ Note that the original wording mentioned FRF 1,000,000. In 1998, the exchange rate was 1 USD per 5.9 FRF.
} 
models, life-cycle models when the change in mortality lasts over an infinitesimally short time (Johansson 2003) as well as wage-risk trade-off models for marginal changes in risk (see Rosen 1988, Viscusi 1993) rely on the EU theory and express the VPF as a marginal rate of substitution between wealth and risk of death. What happens if this approach is crudely applied to the results of the above experiment?

Before answering, it should be pointed out that studies aiming at valuing life never ask the kind of direct question we use. They generally use either data from market choices that involve an implicit trade-off between risk and money (labor or housing markets, transportation, self-protection or averting behaviors), or stated preferences elicited in more subtle ways and using unidentified victims. ${ }^{2}$ Moreover, stated preferences suffer from limitations, both generally and in this case: the actual behavior is not observed; due to incorrect sensitivity to probabilities, smaller changes in risk tend to induce higher VPF estimates (Beattie et al. 1998); a significant gap exists between willingness to pay and willingness to accept...

Finally, the lack of monetary incentives in this experiment may puzzle the reader and is briefly justified below. A number of authors (e.g. Smith 1976, Harrison 1994, or Smith and Walker 1993) emphasize the importance of paying subjects in real cash and providing appropriate monetary incentives in experiments, based on the principle that monetary incentives are needed to motivate people sufficiently when answering hypothetical questions and that this leads to better performance. On the contrary, other authors, including social (and economic) psychologists (Slovic, 1969; Loewenstein, 1999; Tversky and Kahneman, 1992), consider that subjects should be intrinsically motivated enough to answer truthfully in the experiment and that social or affective incentives may be even better motivators than monetary incentives.

This is a controversial issue among researchers, regularly raised by new experiments or

\footnotetext{
${ }^{2}$ However, in our experiment, the victim, although identified, is only exposed to an (infinitesimal) risk change, not to certain death.
} 
meta-analyses. A case in point is Camerer and Hogart (1999), who analyzed 74 experiments either known to them (1953-1998) or published in famous US journals from 1990-98. These studies all varied incentives substantially. The authors found no effect on mean performance in most of the studies (though variance is usually reduced by higher payment) and noted that "no replicated study has made rationality violations disappear purely by raising incentives". They conclude that apart from cases in which subjects are required to make a major cognitive effort and/or face an incitement to lie, monetary incentives are not mandatory.

Neither of these conditions applies to our experiment, which moreover has several characteristics suggesting that subjects were intrinsically motivated to answer truthfully: they were volunteer colleagues, with a potential reciprocity concern vis-à-vis the experimenter; they were told they would be provided with a summary of the experimental results; the topic can be considered entertaining and of intellectual interest; and the experiment was not time-consuming at all (5 minutes). We are therefore confident that participants answered seriously even without monetary incentives, which would have been difficult to implement in this case.

All that being said, subjects face a choice between compensation $(€ 152,450)$ for accepting a change in risk of death (increase of $10^{-9}$ ) and a status quo alternative. Subjects who answer ' $Y e s$ ' clearly consider that $€ 152,450$ is enough to compensate for the increase in death risk, whereas those who answer ' $N o$ ' do not. Due to the referendum-type elicitation question, the minimum amount at which subjects would accept the increase in risk is unknown. Among the 64 responses collected, 33 subjects answered ' $N o$ ' and 31 answered 'Yes' (see the second column of Table 1 for details by answer type).

\section{[INSERT TABLE 1 ABOUT HERE]}

Do some subjects' characteristics explain such behavior? We look for dependences between the answer given and individual characteristics with contingency chi-square tests (see 
the second column of Table 2). No evidence of dependence is found: the p-values are far from the usual significant levels in use. These results are confirmed by performing an analysis of variance for main effects and crossed effects (interactions): no characteristic appears significantly discriminant in explaining the $Y e s / N o$ answer. It is hence not surprising that these characteristics fail to explain subject answers when used as explanatory variables in binomial discrete choice models (Probit and Logit): the percentage of correct predictions obtained does not differ from what would be obtained by chance!

\section{[INSERT TABLE 2 ABOUT HERE]}

Hence, according to EU predictions, each of the 33 subjects who answers ' $N o$ ', implicitly values his/er own life at more than $€\left(152,450 / 10^{-9}\right)$, that is more that $€ 152.4$ trillion! This is seven times the world's total GNP at the time of the survey ( $€ 20.8$ trillion in 1998, Word Bank, 1999), far from the $\$ 1.7-\$ 7$ million range usually obtained in the literature (see for instance Miller, 2000; Mrozek and Taylor, 2002; Viscusi and Aldy, 2003; or US-EPA, 2004). As a consequence, the overall self-assessed value of the members of this research centre amounts to at least $€ 6.8710^{14}$ !

What is going wrong? If we rule out the possibility that subjects cannot correctly understand low probabilities (all belong to a Quantitative Economics research center and $84 \%$ of them have followed (or given) graduate courses in Statistics), one plausible explanation is that subjects who answer ' $N o$ ' gave no consideration to what 'one chance in 1 billion' means, but rather focussed on the frightening event and disregarded the probability corresponding to this event. We will show in Section 3 that it is because EU is insensitive to rare events that it cannot handle the catastrophic dimension associated with the rare event in this pill experiment. 


\subsection{The 2009 follow-up pill experiment}

In January 2009, the same question was again put by e-mail to the initial 1998 sample as well as to new members of the same Research Center. ${ }^{3}$ Examining the motivations for their answer is crucial, and to this end, they were questioned on the influence various factors had on their answer. Subjects then gave a mark on a scale of 0 to 5 (where ' 0 ' equates to 'no influence at all ' and ' 5 ' equates to 'very strong influence') to the following changes in factors: marital / familial status (Family), financial status (Financial), health status (Health), age (Age), life expectancy (LifeExpec), perception of the probability (PercProba), opinion w.r.t. this type of issue (OpIssue), relation to chance (Chance), relation to death (Death). An open question at the end allowed subjects to state other factors (Other) or give open comments.

Of the 64 initial members, 2 had unfortunately died, it was impossible to find a way of contacting 3 at the time of the study, and 2 did not answer e-mails. The 2009 sample is thus composed of 57 out of $64(89 \%)$ initial members and 63 new members, i.e. a total of 120 subjects. The answers to the pill question were as follows: 77 subjects answered ' $N o$ ' and 43 answered 'Yes' (see third column of Table 1 for descriptive statistics by answer type). Once again, dependences between the answer given (Yes/No) and individual characteristics were tested with contingency chi-square tests (see the third column of Table 2) and, as for the 1998 answers, no evidence of significant dependence was found.

Three interesting questions remain: what motivates 2009 subjects' answers, do subjects give different answers in 1998 and 2009 and why do some subjects answer differently in 1998 and $2009 ?$

We first present in Table 3 sample statistics on the motivations given by subjects, ranked by decreasing mean mark. Note that 17 out of 120 subjects (14.2\%) gave a null mark to

\footnotetext{
${ }^{3}$ The 1998 amount is about $€ 182,000$ in 2009 due to inflation (the exchange rate was 1 USD per $0.77 €$ in 2009).
} 
all motivations (including Other), thus considering that none of them influenced their 2009 answer. Table 3 shows that among the sample, opinion w.r.t. this type of issue (OpIssue), perception of the probability (PercProba) and marital / familial status (Family) seem to have the greatest weight in explaining the answers.

\section{[INSERT TABLE 3 ABOUT HERE]}

We then test whether 1998 subjects answer differently from 2009 subjects. The standard test consists in comparing the proportion of ' $Y e s$ ' (or indifferently ' $N o$ ') in the two samples. However, we should take into account that the samples overlap since 57 subjects belong to both samples. We then use a test of equal proportion that accounts for that (in particular for the fact that the variance of the two proportions are the same under the null hypothesis, see Bland and Butland). The proportion of ' $N o$ ' for the 2009 sample $(\mathrm{n}=120)$ significantly exceeds that for the 1998 sample $(n=64$, two-sample proportion-comparison test with "Bland and Butland" p-value $=.0246)$. If we restrict to the overlapping subjects $(\mathrm{n}=57)$, we obtain the same result (one-sample proportion-comparison t test with "standard" p-value $=.0297)$. Let us consider now the two sub-samples that answer the 2009 follow-up experiment: the 57 subjects that previously answered the 1998 experiment and the 63 new subjects. The proportions of ' $N o$ ' for these two sub-samples do not significantly differ (two-sample proportion-comparison test with "standard" p-value $=.1776$ ).

Finally, let us focus on the 57 subjects that answered both 1998 and 2009 surveys. Moving from the aggregate level to the individual level, we observe that 15 subjects changed their mind between 1998 and 2009: 12 by switching from ' $Y e s$ ' to ' $N o$ ' and 3 from ' $N o$ ' to 'Yes'. Five of them gave open comments to explain what motivates their change "I take much bigger risks in everyday life without such a high reward, so I have decided to change from 'No' to 'Yes'", "I now have two children and do not want to add any additional risk - however tiny it may be - that may have painful implications for their life" ('Yes' to 'No'), "My position on the consequences my death would have for my relatives has 
changed" ('Yes' to 'No'), "I am now married with twins, fully happy and I want nothing more" ('Yes' to 'No') and "the 1998 ratio of gain variation over risk variation expressed in French Francs (i.e., $10^{6} / 10^{-9}$ ) was more attractive than the current ratio expressed in euros $\left(182,000 / 10^{-9}\right)$ w.r.t. the probability perception, even though the monetary gains are similar in terms of purchasing power" (Yes to No). Note that in 9 changes out of 15 , the subject had had one (or several) child(ren) since 1998.

Among these 57 subjects, descriptive statistics by change in answer are shown in the fourth column of Table 1 (with 'Yes' for a change and 'No' for no change). Dependences between a change in answer and individual characteristics were also tested with contingency chi-square tests (see last column of Table 2) and significant dependences were found for gender $(\mathrm{p}$-value $=0.003)$, position $(\mathrm{p}$-value $=0.047)$ and change in parenthood status $(\mathrm{p}$ value $=0.081$. We now turn to a conditional analysis of the determinants of a change in answer by estimating a Logit model. Table 4 presents the best model (Huber/White robust estimator of variance is used) explaining the probability of a change in answer. The overall quality of the model is good, as shown by the strong rejection of the joint nullity of the estimated coefficient ( $\mathrm{p}$-value $<0.01)$, the high Pseudo $\mathrm{R}^{2}(0.364)$ and the percentage of correct predictions (48 out of 57 , i.e. $84.2 \%$ ).

\section{[INSERT TABLE 4 ABOUT HERE]}

Considering now the significant variables, we found that being a female ( $\mathrm{p}$-value $=0.008$ ), being over 50 years old (p-value=0.03) and giving a high mark to the motivation "Family" $(\mathrm{p}$-value $=0.003)$ and "PercProba" ( $\mathrm{p}$-value=0.019) significantly increase the probability of a change. The marginal effect of the explanatory variables on the probability of a change is computed at the sample means and given in the last column of Table 4, as well as the corresponding p-values. Hence, ceteris paribus, being a female instead of a male increases the probability of change by $40 \%$, being older than 50 by $58 \%$, and one additional point in 
the mark given to Family (resp. PercProba) increases the probability of a change by $7 \%$ (resp $5.8 \%)$.

Overall, the pill experiments lead to the following results. First, 52\% of the 1998 sample and $64 \%$ of the 2009 sample implicitly value their own life at more than $€ 152.4$ trillion according to EU predictions. Second, subjects' characteristics are not significant in explaining 1998 or 2009 answers, but being a female and being over 50 years old increase the likelihood of a change in answer between 1998 and 2009 in the sample that answered both surveys. The marital / familial status and the perception of the probability motivations also explain change in answers. Third, because in 9 changes out of 15 the subject had had one (or several) child(ren) since 1998, and because a change in parenthood status is significant in explaining changes in answers, familial status could be seen as the main driver of the answers. However, the perception of (low) probability is certainly also relevant in explaining answers because the 2009 answers of subjects already surveyed in 1998 and those of new subjects do not differ significantly.

Section 3 presents recent contributions to the modeling of catastrophic risks and shows how well these experimental results fit the axiomatic approach introduced by Chichilnisky (2000, 2002).

\section{Why EU theory fails, and a solution}

A close examination of EU theory appears worthwhile, to identify the source of its inability to rationalize about half of subjects' choices among catastrophic and rare outcomes.

\subsection{Notations and Expected Utility}

Uncertainty is described by a system that is in one of several states, indexed by the real numbers with a standard Lebesgue measure. In each state a monotonically increasing continuous utility function $u: R^{n} \rightarrow R$ ranks the outcomes, which are described by 
vectors in $R^{n}$. When the probability associated with each state is given, a description of the utility achieved in each state is called a lottery: a function $f: R \rightarrow R$. Choice under uncertainty means the ranking of lotteries. Bounded utilities are required by Arrow (1971) and many others to avoid the St. Petersburg Paradox (see Chichilnisky, 2000, 2009). An event $E$ is a set of states, and $E^{c}$ is the set of states of the world not in $E$ (i.e. the complement of the set $E$ ).

Axioms for choice under uncertainty describe natural and self-evident properties of choice, like ordering, independence and continuity. These classic axioms were developed half a century ago by von Neumann, Morgenstern, Arrow, Hernstein and Milnor. Continuity is a standard requirement that captures the notion that nearby stimuli give rise to nearby responses, which is reasonable enough. Arrow (1971) calls it Monotone Continuity (MC) and Hernstein and Milnor (1953) call it Axiom 2. ${ }^{4}$ However continuity depends on the notion of 'closeness' that is used. A monotone decreasing sequence of events $\left\{E^{i}\right\}_{i=1}^{\infty}$, is a sequence for which for all $i, E^{i+1} \subset E^{i}$. If there is no state in the world common to all members of the sequence, $\bigcap_{i=1}^{\infty} E^{i}=\varnothing$ and $\left\{E^{i}\right\}$ is called a vanishing sequence. For example, in the case of the real line, the sequence $\{(n, \infty)\}, n=1,2,3 \ldots$, is a vanishing sequence of sets.

In Arrow (1971), two lotteries ${ }^{5}$ are close to each other when they have different consequences in small events, which he defines as "An event that is far out on a vanishing sequence is 'small' by any reasonable standards" and more formally, as follows:

Axiom of Monotone Continuity (MC) Given $a$ and $b$, where $a \succ b$, a consequence $c$, and a vanishing sequence $\left\{E^{i}\right\}$, suppose the sequences of actions $\left\{a^{i}\right\},\left\{b^{i}\right\}$ satisfy the

\footnotetext{
${ }^{4}$ Note that Arrow (1971), p. 257, introduces the axiom of Monotone Continuity attributing it to Villegas (1964), p. 1789. It requires that modifying an action in events of small probabilities should lead to similar rankings. At the same time Hernstein and Milnor (1953) require a form of continuity in their Axiom 2 that is similar to Arrow's Monotone Continuity and leads to their Continuity Theorem on p. 293.

${ }^{5}$ The equivalent to the notion of "lotteries" in our framework is the notion of "actions" in Arrow (1971).
} 
conditions that $\left(a^{i}, s\right)$ yields the same consequences as $(a, s)$ for all $s$ in $\left(E^{i}\right)^{c}$ and the consequence $c$ for all $s$ in $E^{i}$, while $\left(b^{i}, s\right)$ yields the same consequences as $(b, s)$ for all $s$ in $\left(E^{i}\right)^{c}$ and the consequence $c$ for all $s$ in $E^{i}$. Then for all $i$ sufficiently large, $a^{i} \succ b$ and $a \succ b^{i}$.(Arrow 1971, p. 48)

In Arrow's framework, two lotteries that differ in sets of small enough Lebesgue measure are very close to each other.

On the basis of the standard axioms of choice (including MC), a crucial result established that individuals optimize the ranking of lotteries $W_{E U}(f)$ according to an expected utility function. The expected utility of a lottery $f$ is a ranking defined by $W_{E U}(f)=$ $\int_{x \in R} f(x) d \mu(x)$ where $\mu$ is a measure with an integrable density function $\phi($.$) that belongs$ to the space of all measurable and integrable functions on $R$ so $\mu(A)=\int_{A} \phi(x) d x$, where $d x$ is the standard Lebesgue measure on $R$. The ranking $W_{E U}($.$) is a continuous linear$ function that is defined by a countably additive measure $\mu{ }^{6}$

\subsection{Recent contributions to the modeling of catastrophic risks}

In recent work, a catastrophic risk is described as an event that has "a very low probability of materializing, but that if it does materialize will produce a harm so great and sudden as to seem discontinuous with the flow of events that preceded it" (Posner, 2004, p. 6). This interpretation of catastrophic risks is entirely consistent with ours. However, Posner (2004) does not model decisions with catastrophic risks - he refers to EU analysis and points out that this analysis is inadequate to explain the decisions that people make when confronted with catastrophic risks.

The modeling of catastrophic risks in Weitzman (2009) is based on EU and assumes that there are "heavy tails" (defined as distributions that have an infinite moment generating function). He seeks to explain behavioral discrepancies by attributing them to these

\footnotetext{
${ }^{6} \mathrm{~A}$ 'countably additive' measure is defined in Appendix.
} 
unexplained "heavy tails". It should be noted that, "heavy tails" being inconsistent with the main axioms of EU, this leads to the non-existence of a robust solution and unacceptable conclusions, like using all the current resources to mitigate future catastrophes. As noted by Buchholz and Schymura (2010), a model assuming both EU theory and utility function unbounded below leads to catastrophic events playing a dominant role in the decision-making process. In contrast, the choice of utility functions that are bounded below leads to implausibly low degrees of relative risk aversion and catastrophic events playing no role in the decision-making process.

To avoid infinite values, Weitzman (2011) suggests thinning or truncating the probability distribution, or putting a cap on utility. Other authors try to reconcile EU and "heavy tails" by using specific utility functions other than power Constant Relative Risk Aversion utility. For instance, Ikefuji et al. (2010) propose the two-parameter Burr function or exponential utility function and Millner (2011) proposes the (bounded) Harmonic Absolute Risk Aversion function to model individual preferences. However, this approach yields results driven by subjective choices like functional forms or parameter values, which is not fully satisfactory, as Weitzman (2011) admits.

Chichilnisky's (1996, 2000, 2002, 2009) approach, presented hereafter, differs from the above in proposing a systematic axiomatic foundation for modeling catastrophic risks or for making decisions when risks are catastrophic. She shows why EU theory fails to explain half the answers in the experiment and identifies the $\mathrm{MC}$ axiom as the source of the problem, proposing an alternative set of axioms that appears to fit the experimental evidence.

\subsection{The failure of EU and a solution}

The failure of EU appears to be due to the MC axiom which implicitly postulates that rational behavior should be 'insensitive' to rare events with major consequences. More specifically, the culprit is the underlying definition of proximity that is used in the MC 
axiom, where two events are close to each other when they differ on a set of small measure no matter how great the difference in their outcomes. Chichilnisky (2000, 2002, 2009) used a $L_{\infty}$ sup norm that is based on extreme events to define closeness: two lotteries $f$ and $g$ are close when they are uniformly close almost everywhere (a.e.), i.e. when $\sup _{R} \mid$ $f(t)-g(t) \mid<\epsilon$ a.e. for a suitable small $\epsilon>0 .{ }^{7}$ As a consequence, some catastrophic events are small under Arrow's definition but not necessarily under Chichilnisky's.

The core here is that her definition of closeness is more sensitive to rare events than Arrow's. It implies that a probability can be considered as low enough to make the lottery involving death acceptable, depending on what the other outcomes are. This higher sensitivity constitutes the second of her three axioms, which must be satisfied by a ranking $W$ to evaluate lotteries:

\section{Axiom 1: The ranking $W: L_{\infty} \rightarrow R$ is linear and continuous on lotteries.}

The ranking $W$ is called continuous and linear when it defines a linear function on the utility of lotteries that is continuous with respect to the norm in $L_{\infty}$.

\section{Axiom 2: The ranking $W: L_{\infty} \rightarrow R$ is sensitive to rare events.}

A ranking function $W: L_{\infty} \rightarrow R$ is called insensitive to rare events when it neglects low probability events; formally if given two lotteries $(f, g)$ there exists $\epsilon=\epsilon(f, g)>0$, such that $W(f)>W(g)$ if and only if $W\left(f^{\prime}\right)>W\left(g^{\prime}\right)$ for all $f^{\prime}, g^{\prime}$ satisfying $f^{\prime}=f$ and $g^{\prime}=g$ a.e. on $A \subset R$ when $\mu\left(A^{c}\right)<\epsilon$. We say that $W$ is sensitive to rare events, when $W$ is not insensitive to low probability events.

\section{Axiom 3: The ranking $W: L_{\infty} \rightarrow R$ is sensitive to frequent events.}

\footnotetext{
${ }^{7}$ A similar topology was used in Debreu's (1953) formulation of Adam Smith's Invisible Hand theorem.
} 
Similarly, $W: L_{\infty} \rightarrow R$ is said to be insensitive to frequent events when for every two lotteries $f, g$ there exists such $\epsilon(f, g)>0$ that $W(f)>W(g)$ if and only if $W\left(f^{\prime}\right)>W\left(g^{\prime}\right)$ for all $f^{\prime}, g^{\prime}$ such that $f^{\prime}=f$ and $g^{\prime}=g$ a.e. on $A \subset R: \mu\left(A^{c}\right)>1-\epsilon$. We say that $W$ is sensitive to frequent events when $W$ is not insensitive to frequent events.

Our notion of 'nearby' is stricter and requires that the lotteries be close almost everywhere, which implies sensitivity to rare events. Chichilnisky (2009) proved that EU theory fails to explain the behavior of individuals facing catastrophic events since:

\section{Theorem 1: A ranking of lotteries $W(f): L_{\infty} \rightarrow R$ satisfies the Monotone Con-} tinuity Axiom if and only if it is insensitive to rare events. (see proof in Chichilnisky 2009).

A formal statement of the theorem is hence $M C \Leftrightarrow \neg$ Axiom 2 . The simple example below shows why the axiom MC leads to insensitivity to rare events.

Example: Assume that the Axiom MC is satisfied. By definition, this implies for every two lotteries $f \succ g$, every outcome $c$ and every vanishing sequence of events $\left\{E^{i}\right\}$ there exists $N$ such that arbitrarily altering the outcomes of lotteries $f$ and $g$ on event $E^{i}$, where $i>N$, does not alter the ranking, namely $f^{\prime} \succ g^{\prime}$, where $f^{\prime}$ and $g^{\prime}$ are the altered versions of lotteries $f$ and $g$ respectively. ${ }^{8}$ In particular since, for any given $f$ and $g$, Axiom $M C$ applies to every vanishing sequence of events $\left\{E^{i}\right\}$, we can choose a sequence of events consisting of open intervals $I=\left\{I^{i}\right\}_{i=1}^{\infty}$ such that $I^{i}=\{x \in R: x>i\}$ and another $J=\left\{J^{i}\right\}_{i=1}^{\infty}$ such that $J^{i}=\{x \in R: x<-i\}$. Consider the sequence $K=\left\{K^{i}\right\}$ where $K^{i}=I^{i} \cup J^{i}$. The sequence $K$ is a vanishing sequence by construction. Therefore there exists an $i>0$ such that for all $N>i$, any alterations of lotteries $f$ and $g$ over $K^{N}$, denoted

\footnotetext{
${ }^{8}$ For simplicity, we consider alterations in those lotteries that involve the 'worst' outcome $c=\inf _{R}$ | $f(x), g(x) \mid$, which exists because $f$ and $g$ are bounded a.e. on $R$ by assumption.
} 
$f^{N}$ and $g^{N}$ respectively, leave the ranking unchanged i.e. $f^{N} \succ g^{N}$. Therefore Axiom $M C$ implies insensitivity of ranking $W$ in unbounded sets of events such as $\left\{E^{i}\right\}$.

Hence, because EU (and more generally approaches relying on the EU axiomatic) relies on the MC Axiom that considers two lotteries to be close when they differ in events of small measure, it is insensitive to rare events. ${ }^{9}$ This leads directly to the way of valuing life we describe above: measured by the low probability of death acceptable in exchange for an extra amount of money. This classic axiom postulates that rational behavior should be 'insensitive' to rare events with major consequences such as death, as proved in Theorem 1. This insensitivity underestimates our true responses to catastrophes, creating an impression of irrationality from observed behavior that is not fully justified. Taking a family of subsets of events containing no rare events, for example when the Lebesgue measure of all events contemplated is bounded below, EU satisfies all three Axioms. Indeed, in the absence of rare events, Axiom 2 is an empty requirement and Axioms 1 and 3 are consistent with EU theory.

The MC axiom is strong and somewhat counterintuitive, so it is not surprising that the experimental results contradict it. It requires, for example, that the measures of a nested sequence of intervals of events $\{x: x>n\}$ for $n=1,2, \ldots$ decrease all the way to zero. This zero limit is required even though the intervals themselves $\{x: x>n\}$ are all essentially identical and could, for example, be expected to have the same measure, at least in some cases. No explanation is provided for this somewhat unusual and strong axiom, in which essentially a sequence of identical sets is assumed always to have measures converging to zero.

In contrast, the axioms for decision-making with catastrophic risks utilized here require sensitivity to rare events, a new axiom which applies in some cases and not in others. This new axiom allows for the measures in such sequences converging to zero in some cases and

\footnotetext{
${ }^{9}$ See also Chichilnisky $(1996,2000,2002)$ for a general proof that EU is insensitive to rare events.
} 
not in others, a logical negation of MC. This flexibility seems more intuitive and plausible. While the universal applicability of MC is ruled out, a different type of continuity is required in the new axioms. Experimental evidence contradicts EU theory when catastrophes are involved (as Posner, 2004, explains himself), and therefore it contradicts the universal applicability of its underlying axiom, MC. The experimental results presented in this article are consistent with the new axiom of sensitivity to rare events. The difference between MC and sensitivity to rare events is at the basis of the new form of decision-making under uncertainty involving catastrophic risks introduced and developed in Chichilnisky (1996, 2000, 2002, 2009), and can be viewed as the axiomatic foundation for the experimental results presented in this article.

In the experiments presented here, the value of life can be considered as clearly defined if a subject is willing to accept the same probability of death in exchange of the same amount of money and the value of life is therefore contingent on the amount itself. Our findings show, however, that for the same amount of money, the subject may or may not be willing to accept the same small probability of death, which indicates that subjects are taking into consideration factors other than the amount of money and the probability of death when making a decision. This appears to conflict with existing theory based on the MC axiom, where the probability of death a subject is prepared to accept can be clearly defined for any given payment. In particular we establish the following result:

Theorem 2: A ranking of lotteries $W: L_{\infty} \rightarrow R$ that satisfies Axiom 2 (i.e. that is sensitive to rare events) determines a value of life that changes depending on outcomes other than the amount of money.

Proof: Theorem 1 showed that sensitivity to rare events is the negation of the MC Axiom. This implies that for two given lotteries $f \succ g$, every outcome $c$ and every vanishing sequence of events $\left\{E^{i}\right\}$ there exists $N$ such that arbitrarily altering the outcomes of lotteries $f$ and $g$ on event $E^{i}$, where $i>N$, does not alter the ranking, namely $f^{\prime} \succ g^{\prime}$, where $f^{\prime}$ and $g^{\prime}$ 
are the altered versions of lotteries $f$ and $g$ respectively, while for some other lotteries $f \succ$ $g$, every outcome $c$ and some vanishing sequence of events $\left\{E^{i}\right\}$ there exists $N$ such that arbitrarily altering the outcomes of lotteries $f$ and $g$ on event $E^{i}$, where $i>N$, does alter the ranking, namely $g^{\prime} \succ f^{\prime}$, where $f^{\prime}$ and $g^{\prime}$ are the altered versions of lotteries $f$ and $g$ respectively. Recall that - as in Theorem 1 above - we have considered alterations in the lotteries that involve the 'worst' outcome $c=\inf _{R}|f(x), g(x)|$. The worst outcome can be identified with "death", and is common to the two sets of lotteries under comparison. The alterations in both cases are the same, representing small enough probabilities of death. This implies that, for a small enough probability of death (determined by $N$ ) in the first two lotteries, the subject will accept the risk of death when offered the small payment, while in the second lottery the same probability of death (represented by $N$ ) is not small enough. In other words, depending on other outcomes of the lotteries, the subject will accept a small probability of death, or will not accept that probability. Therefore the value of life depends on other outcomes of the lottery, as we wished to establish.

Do some decision criteria satisfy all three Axioms in the presence of rare events? Yes, if we modify EU by adding another component called 'purely finitely additive' elements of $L_{\infty}^{*}{ }^{10}$ that embodies the notion of sensitivity for rare events. The only acceptable rankings $W$ under the three axioms above are a convex combination of $L_{1}$ function plus a purely finitely additive measure putting all weight on extreme or rare events, as stated in the Theorem below:

Theorem 3: A ranking of lotteries $W: L_{\infty} \rightarrow R$ satisfies all three axioms 1, 2 and 3, if and only if there exist two continuous linear functions on $L_{\infty}, \phi_{1}$

\footnotetext{
${ }^{10}$ The space $L_{\infty}^{*}$ is called the 'dual space' of $L_{\infty}$, and is known to contain two different types of rankings $W($.$) , (i) integrable functions in L_{1}(R)$ that can be represented by countably additive measures on $R$, and (ii) 'purely finitely additive measures' which are not representable by functions in $L_{1}$ (Chichilnisky, 2000), and are not continuous with respect to the Lebesgue measure of $R$. See Appendix for a definition of a 'finitely additive' measure.
} 
and $\phi_{2}$ and a real number $\lambda, 0<\lambda<1$, such that:

$$
W_{T F A}(f)=\lambda \int_{x \in R} f(x) \phi_{1}(x) d x+(1-\lambda)\left\langle f, \phi_{2}\right\rangle
$$

where $\int_{R} \phi_{1}(x) d x=1$, while $\phi_{2}$ is a purely finitely additive measure. (see proof in Chichilnisky 1996, 2000, 2002).

The intuition behind this Theorem is that the first term of the utility in (??) is akin to $\mathrm{EU}$, and therefore introduces a measure of sensitivity to normal or relatively frequent events. The density $\phi_{1}(x)$ defines a countably additive measure that is absolutely continuous with respect to the Lebesgue measure. ${ }^{11}$

The second term of the utility in (??) is inconsistent with the MC axiom, and satisfies a different type of continuity, under a topology called "The Topology of Fear" (Chichilnisky, 2009). This second term is very sensitive to rare events and balances out the first term in the characterization, which is only sensitive to normal or frequent events. The operator $\left\langle f, \phi_{2}\right\rangle$ represents the action of a measure $\phi_{2} \epsilon L_{\infty}^{*}$ that differs from the Lebesgue measure in placing full weight on rare events. Remember that $\phi_{2}$ cannot be represented by an $L_{1}$ function.

The two terms together therefore satisfy both 'sensitivity to rare events', and 'sensitivity to frequent events', as is required by the new axiomatic treatment of decision making under uncertainty with catastrophic risks used here. The implied decision criteria that emerge from the new axioms are a combination of EU with extreme responses (to extreme events like death), and seem more in line with experimental evidence.

Indeed, it seems that purely finitely additive measures could play an important role in explaining how our brains respond to extreme risks. When the number of choices is finite there is a simpler way to explain the criterion of choice: it is similar to a convex

\footnotetext{
${ }^{11} \mathrm{~A}$ measure is called absolutely continuous with respect to the Lebesgue measure when it assigns zero measure to any set of Lebesgue measure zero; otherwise the measure is called singular.
} 
combination of EU and a maximin. EU is optimized while at the same time avoiding those choices that involve catastrophic outcomes, such as death. This rule is inconsistent with EU and will rank a choice that involves death much lower than EU would. Therefore any observer that anticipates EU optimization will be disappointed, and will believe that there is irrationality. ${ }^{12}$ But this is not true, as the rule becomes rational once we take into account rational responses to extreme events. It is consistent with what people do on an everyday basis, with what is observed in the experiments presented here and also with what Arrow's famous comment implies.

\section{Discussion and concluding remarks}

How the Axiom MC creates insensitivity to rare events can be illustrated by the following situation used by Arrow (1966) to show how people value their lives, along the same lines as the discussion in the Introduction. If $a$ is an action that involves receiving one cent, $b$ is another that involves receiving zero cents, and $c$ is a third action involving receiving one cent and facing a low probability of death, Arrow's Monotone Continuity requires that the third action involving death and one cent should be preferred to the second involving zero cents when the probability of death is low enough. Even Arrow says of his requirement 'this may sound outrageous at first blush...' (Arrow, 1966, p. 256, 1. 28-29). Outrageous or not, we saw in Theorem 1 that MC leads to the neglect of rare events with major consequences like death.

Theorem 1 shows that Axiom 2 eliminates those examples that Arrow calls outrageous. We can also see how Axiom 2 provides a reasonable solution to the problem, as follows. Axiom 2 implies that there are catastrophic outcomes, such as the risk of death, so terrible

\footnotetext{
${ }^{12}$ Note that EU could be used in certain cases to rationalize answers like the one we obtained, without providing a consistent set of axioms that create a well defined theory, by assuming that some subjects are infinitely averse to risk (unbounded below utility function). However, this is a somewhat ad hoc treatment not satisfactory theoretically since it brings back in the St Petersburg Paradox.
} 
that people are unwilling to accept a low probability of death to obtain one cent versus 0 cents, no matter how low that probability may be. Indeed, according to the sensitivity Axiom 2, no probability of death is acceptable when one cent and 0 cents are involved. However according to Axiom 2, in some cases, the probability can be low enough to make the lottery involving death acceptable. As shown in Theorem 2, it all depends on what the other outcomes are. This becomes clear in our approach and seems a reasonable solution to the problem that Arrow raises.

For example, if in the above example "one cent were replaced by one billion dollars" as Arrow (1966 p. 256, lines 31-32) suggests - under certain conditions we may be willing to choose the lottery that involves a low probability of death and one billion dollars over the lottery that offers 0 cents. ${ }^{13}$ Indeed, some of the subjects in the pill experiment state that they might have chosen to take the pill for a larger amount ("the amount is not big enough" (3 subjects), "the amount is too small to dramatically change my life", "I would have answered 'Yes' if I was almost in absolute poverty").

More to the point, consider the same type of death risk: a low probability of death caused by a medicine that can cure an otherwise incurable cancer may be preferable to no cure. A sick person may evaluate a cure - no matter how risky - higher than a healthy person would, and may be willing to take risks that a healthy person would not. In the same spirit, as shown in the pill experiment, the same individual may change his/er mind depending on factors exogenous to the outcomes. Here, among the two reasons that significantly explained such a change in the subjects that answered both 1998 and 2009 surveys, were "change in marital/familial status" and "change in the perception of the probability".

The former has to do with the painful implications the death would have for relatives. The latter has to do with the subjective perception of the probability $10^{-9}$. One reason

\footnotetext{
${ }^{13}$ Or if the death event had been replaced by a less frightening event, say a $€ 152,450$ loss, we are willing to bet that most of the subjects in the pill experiment would have accepted the $10^{-9}$ probability of loss.
} 
suggests itself: the 9/11 attacks, which occurred between the two experiments. Indeed, although subjects may have considered the outcome "simultaneous crashes of two commercial flights into the World Trade Center within the same hour" of tiny probability, the fact that it actually occurred may have led them to change their perception of tiny probability. No catastrophe had ever been so widely covered worldwide. This may explain that, in 2009 answers, new subjects do not significantly differ from previously 1998 surveyed subjects when surveyed in 2009. Indeed, Sunstein (2003) also provides evidence that individuals show unusually strong reactions to low-probability catastrophes especially when their emotions are intensely engaged. This "probability neglect" is also explored in Sunstein and Zeckhauser (2011) regarding both fearsome risks and the resulting damaging overreactions shown in individual behavior and government regulations.

Recently, Chanel and Chichilnisky (2009) report experimental results from a study of the predictions of the standard EU framework under catastrophic risks. Subjects faced choices among events involving "being locked up in a room with no chance of escaping, being freed or communicating (with relatives, friends...), with nothing interesting to do". The events differed as to the duration of detention, and the catastrophic event was created by making the period of detention 40 years. Interestingly, the results obtained are in line with that obtained here: more than half the subjects did not behave according to EU theory whereas the remaining half answered according to EU theory; however all behaved according to the approach proposed in this article.

To conclude, the alternative approach proposed in this article furthers the treatment of catastrophic outcomes in two ways.

First, it provides a new measure for the value of life with two important characteristics: it values life more highly than under the EU criterion, and this value is shown to depend on other factors, not only on the numerical value of what is being offered. This is because catastrophes are worst-case events whose weight depends on what else is going on in people's 
lives.

Second, the alternative approach challenges the belief that EU properly expresses rationality in situations involving catastrophic outcomes. Indeed, EU theory is found to perform poorly in explaining the actual behavior of most of the subjects in the experiment, even though these subjects are fully familiar with the logic behind EU theory, while the alternative approach performs quite well. We do not reject the MC axiom outright nor do we reject EU outright. Rather, we find that it is more realistic and satisfactory that $\mathrm{MC}$ be satisfied in some cases and not in others - EU axioms therefore being satisfied in some cases and not in others. Requiring that $\mathrm{MC}$ be satisfied in all cases and thus that EU utility axioms hold in all cases is problematic, since it implies insensitivity to rare and potentially catastrophic events. Hence, our approach stands as an alternative proposal for defining rational behavior in the face of catastrophes such as death. In any case, in the absence of rare events with major consequences, our theory of choice under uncertainty is consistent with and mirrors the standard EU theory (our Axiom 2 is indeed void of meaning), and can therefore be viewed as an extension of classic decision theory.

Finally, an interesting avenue for future research might be to explore how the brain works while considering outcomes involving catastrophic and non-catastrophic events. Are the same zones activated? In the same order? For the same length of time? Functional magnetic resonance imaging or positron emission tomography should help answer these questions, since neuroeconomic decision science is no longer a utopian concept (see for instance Smith et al., 2002; or Knutson and Peterson, 2005).

\section{Acknowledgments}

This research is part of Columbia's Program on Information and Resources, and the Columbia Consortium for Risk Management (CCRM). It was motivated by Olivier Chanel's experimental research and is based on earlier results in "The Topology of Fear" 
(Chichilnisky, 2000, 2002, 2009). It is partly supported by program Riskemotion (ANR-08RISKNAT-007-01), which is gratefully acknowledged. We thank two anonymous referees, Marjorie Sweetko and Jean-Christophe Vergnaud for helpful comments and suggestions, congress participants at Montréal 2010 WCERE for helpful discussions, and the 127 former and current Greqam members for their kind participation.

\section{References}

Allais, M. 1953. Le comportement de l'homme rationnel devant le risque: critique des postulats et axiomes de l'école américaine. Econometrica. 21, 503-546.

Arrow, K., 1966. Exposition of the theory of choice under uncertainty. Synthese. 16, 253-269.

Arrow, K., 1971. Essays in the Theory of Risk Bearing, North Holland, Amsterdam.

Beattie, J., Chilton, S., Cookson, R., Covey, J., Hopkins, L., Jones-Lee, M., Loomes, G., Pidgeon, N., Robinson, A., Spencer, A, 1998. Valuing health and safety controls: A literature review, Report, London: HSE Books.

Bland, J.M., Butland, B.K. Comparing proportions in overlapping samples. Unpublished paper, http://www-users.york.ac.uk/ mb55/overlap.pdf, accessed on 10/24/2011, 13 p.

Buchholz, W., Schymura, M., 2010. Expected utility theory and the tyranny of catastrophic risks. ZEW Discussion Paper No. 10-059, Mannheim.

Camerer, C., Hogarth, R., 1999. The effects of financial incentives in experiments: a review and capital-labor-production framework. J. Risk Uncertainty. 19(1), 7-42.

Chanel, O., Chichilnisky, G., 2009. The influence of fear in decisions: experimental evidence. J Risk Uncertainty. 39, 271-298. 
Chichilnisky, G., 1996. Updating Von Neumann Morgenstern Axioms for Choice under Uncertainty, Proceedings of a Conference on Catastrophic Risks, The Fields Institute for Mathematical Sciences, Toronto Canada.

Chichilnisky, G., 2000. An axiomatic approach to choice under uncertainty with catastrophic risks. Resource Energy Econom. 22, 221-231.

Chichilnisky, G., 2002. Catastrophic risk, in: El-Shaarawi, A., Piegorsch, W. (Eds.), Encyclopedia of Environmetrics. John Wiley \& Sons, Ltd., Chicester: UK, 1 pp. 274-279.

Chichilnisky, G., 2009. The topology of fear. J Mathem. Econom. 45, 807-816.

Debreu, G., 1953. Valuation equilibrium and Pareto optimum. Proceedings of the National Academy of Sciences. 40, 588-592.

Ellsberg, D., 1961. Risk, ambiguity, and the Savage axioms. Quart. J. Econom. 75, 643-669.

Harrison, G., 1994. Expected utility theory and the experimentalists. Empirical Econom. $19,223-253$.

Hernstein, N., Milnor, J., 1953. An axiomatic approach to measurable utility. Econometrica. $21,291-297$.

Ikefuji, M., Laeven, R.J.A., Magnus, J.R., Muris, C., 2010. Expected utility and catastrophic risk in a stochastic economy-climate model. Working Paper 2010-122, Tilburg University, ISSN 0924-7815.

Johansson, P.-O., 2003. The value of a statistical life: Theoretical and empirical evidence. Applied Health Econom. Health Policy Special Issue, 25-33.

Knutson, B., Peterson, R., 2005. Neurally reconstructing expected utility. Games Econom. Behavior. 52, 305-315. 
LeDoux, J., 1996. The Emotional Brain, Simon and Schuster, New York.

Loewenstein, G., 1999. Experimental Economics from the Vantage-Point of behavioural economics. Econom. J. 109, 25-34.

Miller, T., 2000. Variations between Countries in Value of Statistical Life. J. Transport Econom. Policy. 34(2), 169-188.

Millner A., 2011. On welfare frameworks and catastrophic climate risks. Mimeo University of California, Berkley, 29 p., http://ssrn.com/abstract=1799481, accessed on $10 / 24 / 2011$.

Mrozek, J., Taylor, L., 2002. What Determines the value of a life? A meta-analysis. J. Policy Anal. Management. 21(2), 253-270.

Posner, R., 2004. Catastrophe: Risk and response, Oxford University Press, New York.

Rosen, S., 1988. The value of changes in life expectancy. J. Risk Uncertainty. 1(3), 285-304.

Slovic, P., 1969. Differential effects of real versus hypothetical payoffs on choices among gambles. J. Experimental Psych. 80, 434-437.

Smith, K., Dickhaut, J., McCabe, K., Pardo, J.V., 2002. Neuronal substrates for choice under ambiguity, risk, gains and losses. Management Sci. 48, 711-718.

Smith, V., 1976. Experimental economics induced value theory. Amer. Econom. Rev. $66(2), 274-279$.

Smith, V., Walker, J., 1993. Monetary rewards and decision cost in experimental economics. Econom. Inquiry. 31, 245-261.

Sunstein, C., 2003. Terrorism and probability neglect. J. Risk Uncertainty. 26(2/3), $121-136$. 
Sunstein, C., Zeckhauser, R., 2011. Overreaction to fearsome risks. Env. Res. Econ. $48(3), 435-449$.

Tversky, A., Kahneman, D., 1992. Advances in prospect theory: Cumulative Representation of Uncertainty. J. Risk Uncertainty. 5, 297-323.

U.S. Environmental Protection Agency. 2004. Benefits of the Proposed Inter-State Air Quality Rule. Report EPA 452-03-001, January.

Villegas, C., 1964. On quantitative probability $\sigma-$ algebras. Annals Math. Stat. 35, 1789-1800.

Viscusi, K., 1993. The value of risks to life and health. J. Econom. Literature. XXXI, 1912-1946.

Viscusi, K., Aldy, J., 2003. The value of a statistical life: A critical review of market estimates throughout the world. J. Risk Uncertainty. 27(1), 5-76.

Weitzman, M.L., 2009. On modelling and interpreting the economics of catastrophic climate Change. Rev. Econom. Stat. 91(1), 1-19.

Weitzman, M.L., 2011. Fat-tailed uncertainty in the economics of Catastrophic climate Change. Rev. Env. Econom. Policy. 5(2), 275-292.

World Bank. 1999. World Development Report 1998/99, Oxford University Press.

Yosida, K., 1974. Functional Analysis, 4th edition, Springer Verlag, New York Heidelberg.

Yosida, K., Hewitt, E., 1952. Finitely level independent measures. Transactions of the American Mathematical Society. 72, 46-66. 


\section{Appendix: Countably and purely finitely additive measures}

The space of continuous linear functions on $L_{\infty}$ is a well known space called the "dual" of $L_{\infty}$, and is denoted $L_{\infty}^{*}$. This dual space has been fully characterized e.g. in Yosida and Hewitt (1952) or Yosida (1974). Its elements are defined by integration with respect to measures on $R$. The dual space $L_{\infty}^{*}$ consists of $(i) L_{1}$ functions $g$ that define countably additive measures $\mu$ on $R$ by the rule $\mu(A)=\int_{A} g(x) d x$ where $\int_{R}|g(x)| d x<\infty$ and therefore $\mu$ is absolutely continuous with respect to the Lebesgue measure, namely it gives measure zero to any set with Lebesgue measure zero, and (ii) a 'non - $L_{1}$ part' consisting of purely finitely additive measures $\rho$ that are 'singular' with respect to the Lebesgue measure and give positive measure to sets of Lebesgue measure zero; these measures $\rho$ are finitely additive but they are not countably additive. A measure $\eta$ is called finitely additive when for any family of pairwise disjoint measurable sets $\left\{A_{i}\right\}_{i=1, \ldots N} \eta\left(\bigcup_{i=1}^{N} A_{i}\right)=\sum_{i=1}^{N} \eta\left(A_{i}\right)$. The measure $\eta$ is called countably additive when for any family of pairwise disjoint measurable sets $\left\{A_{i}\right\}_{i=1, \ldots \infty} \eta\left(\bigcup_{i=1}^{\infty} A_{i}\right)=\sum_{i=1}^{\infty} \eta\left(A_{i}\right)$. The countably additive measures are in a oneto-one correspondence with the elements of the space $L_{1}(R)$ of integrable functions on $R$. However, purely finitely additive measures cannot be identified by such functions. Yet purely finitely additive measures play an important role, since they ensure that the ranking criteria are 'sensitive to rare events' (Axiom 2). These measures define continuous linear real valued functions on $L_{\infty}$, thus belonging to the dual space of $L_{\infty}$ (Yosida 1974), but cannot be represented by functions in $L_{1}$. 
Table 1 Composition of the samples (in \%)

\begin{tabular}{|c|c|c|c|c|c|c|}
\hline \multirow[t]{3}{*}{ Sample } & \multicolumn{2}{|c|}{1998 survey $(n=64)$} & \multicolumn{2}{|c|}{2009 survey $(n=120)$} & \multicolumn{2}{|c|}{$1998 \& 2009$ survey $(\mathrm{n}=57)$} \\
\hline & \multicolumn{2}{|c|}{ Answers } & \multicolumn{2}{|c|}{ Answers } & \multicolumn{2}{|c|}{ Answer changed? } \\
\hline & No $(\mathrm{n}=33)$ & Yes $(\mathrm{n}=31)$ & No $(\mathrm{n}=77)$ & Yes $(n=43)$ & No $(n=42)$ & Yes $(n=15)$ \\
\hline \multicolumn{7}{|l|}{ Gender } \\
\hline Male & 69.70 & 67.74 & 63.63 & 74.42 & 76.19 & 33.33 \\
\hline Female & 30.30 & 32.26 & 36.37 & 25.58 & 23.81 & 66.66 \\
\hline \multicolumn{7}{|l|}{ Position } \\
\hline Ph.D. student & 42.42 & 35.48 & 37.66 & 51.16 & 33.33 & 46.66 \\
\hline Adm. Staff & 9.10 & 22.58 & 15.58 & 6.98 & 9.52 & 33.33 \\
\hline Ass. prof. /Jr Res. fellow & 24.24 & 19.35 & 16.88 & 23.26 & 30.96 & 6.66 \\
\hline Professor /Sr Res. fellow & 24.24 & 22.58 & 29.88 & 18.60 & 26.19 & 13.33 \\
\hline \multicolumn{7}{|l|}{ Age } \\
\hline 20-29 years old & 36.37 & 41.94 & 36.36 & 53.49 & 30.96 & 53.33 \\
\hline 30-39 years old & 30.30 & 29.03 & 24.68 & 23.25 & 35.71 & 26.66 \\
\hline 40-49 years old & 24.24 & 16.13 & 27.27 & 11.63 & 26.19 & 6.66 \\
\hline Over 50 years old & 9.09 & 12.90 & 11.69 & 11.63 & 7.14 & 13.33 \\
\hline \multicolumn{7}{|l|}{ Net individual income } \\
\hline$<€ 1500 /$ month & 42.42 & 54.84 & 41.56 & 58.14 & 40.47 & 66.66 \\
\hline$€ 1500-€ 2499$ / month & 21.21 & 16.13 & 18.18 & 11.63 & 21.43 & 41.40 \\
\hline$€ 2500-€ 3499$ / month & 24.24 & 9.68 & 15.58 & 13.95 & 23.81 & 0.00 \\
\hline$>€ 3500 /$ month & 12.12 & 19.35 & 24.68 & 16.28 & 14.29 & 35.19 \\
\hline \multicolumn{7}{|l|}{ Parenthood } \\
\hline No children & 60.61 & 61.29 & 29.87 & 37.21 & $62.38^{a}$ & $26.67^{a}$ \\
\hline At least one child & 39.39 & 38.71 & 70.13 & 62.79 & $47.62^{a}$ & $73.33^{a}$ \\
\hline
\end{tabular}

${ }^{a}$ Change in Parenthood between 1998 and 2009. 
Table 2 Contingency chi-square tests (p-values)

\begin{tabular}{lccc}
\hline \hline & Answers in & Answers in & Changes in answers \\
& $1998(\mathrm{n}=64)$ & $2009(\mathrm{n}=120)$ & $1998 \rightarrow 2009(\mathrm{n}=57)$ \\
\hline Gender & 0.866 & 0.822 & 0.003 \\
Position & 0.513 & 0.176 & 0.047 \\
Age & 0.832 & 0.150 & 0.261 \\
Individual income (EUR) & 0.344 & 0.344 & 0.158 \\
Parenthood & 0.955 & 0.413 & 0.081 \\
\hline \hline
\end{tabular}


Table 3 Descriptive statistics on $\operatorname{mark}^{a}$ by motivation $(n=120)$

\begin{tabular}{lccccc}
\hline \hline Motivation & Mean & Std.-Dev. & Minimum & Maximum & \# Non null \\
\hline OpIssue & 2.15 & 2.14 & 0 & 5 & 68 \\
PercProba & 2.01 & 2.21 & 0 & 5 & 60 \\
Family & 1.83 & 2.13 & 0 & 5 & 56 \\
Death & 1.75 & 2.07 & 0 & 5 & 58 \\
Financial & 1.63 & 1.85 & 0 & 5 & 64 \\
Chance & 1.21 & 1.84 & 0 & 5 & 43 \\
Age & 1.09 & 1.70 & 0 & 5 & 43 \\
LifeExpec & 1.01 & 1.62 & 0 & 5 & 40 \\
Health & .82 & 1.43 & 0 & 5 & 37 \\
Other & 4.62 & 0.74 & 3 & 5 & 17 \\
\hline \hline
\end{tabular}

${ }^{a}$ Mark on a scale of 0 (no influence at all) to 5 (very strong influence).

${ }^{b} 17$ subjects express another motivation and state it. 
Table 4 Estimation of the probability of change $(n=57)$

\begin{tabular}{lcccc}
\hline \hline Variable & Estimate & Robust p-value & Marg. effect & Robust p-value \\
\hline Intercept & -3.954 & .000 & - & - \\
Female $(=1)$ & 2.386 & .008 & .396 & .007 \\
Older than $50(=1)$ & 2.815 & .030 & .583 & .018 \\
Family (0-5) & 0.524 & .003 & .070 & .022 \\
PercProba (0-5) & 0.431 & .019 & .058 & .026 \\
\hline LRI / Pseudo R & & 0.3640 & \\
Wald test of joint nullity (p-value) & & $15.41(.0039)$ & \\
Percentage of correct predictions & & $84.2 \%$ & \\
\hline \hline
\end{tabular}

